\title{
Raman and modulated-reflectivity spectra of a strained pseudomorphic ZnTe epilayer on InAs under pressure
}

\author{
Robert J. Thomas, Mark S. Boley, H. R. Chandrasekhar, and Meera Chandrasekhar \\ Department of Physics and Astronomy, University of Missouri, Columbia, Missouri 65211 \\ C. Parks and A. K. Ramdas \\ Department of Physics, Purdue University, West Lafayette, Indiana 47907 \\ J. Han, M. Kobayashi, and R. L. Gunshor \\ School of Electrical Engineering, Purdue University, West Lafayette, Indiana 47907
}

(Received 1 June 1993; revised manuscript received 30 August 1993)

\begin{abstract}
The piezomodulated-, electromodulated-, and photomodulated-reflectivity spectra of a pseudomorphic ZnTe epilayer, grown on an InAs epilayer by molecular-beam epitaxy, exhibit heavy- and light-hole excitonic signatures split by the lattice mismatch induced biaxial compressive strain. This splitting in the pseudomorphic epilayer is studied as a function of applied hydrostatic pressure using photomodulated reflectance spectroscopy at $80 \mathrm{~K}$. With increasing hydrostatic compression, the compressive strain is progressively compensated by the pressure-induced tensile strain. At $\sim 55$ kbars the epilayer becomes strain-free, and is under a biaxial tension at higher pressures. The separation between the heavy- and light-hole signatures is superlinear in pressure, suggestive of a strain or volume deformation-dependent shear deformation-potential constant. We also compare the pressure dependence of the Raman LO phonon of the $\mathrm{ZnTe}$ epilayer on InAs with that of a bulk ZnTe sample at $13 \mathrm{~K}$. The pressure-dependent strain is found to be linear. Accurate values of the first-order strain derivatives of the LO phonons and mode Grüneisen constants are obtained.
\end{abstract}

\section{INTRODUCTION}

Stimulated by their applications in optoelectronic devices with response in the blue such as light-emitting diodes and lasers, ${ }^{1,2}$ extensive studies have been conducted on the electronic and vibrational properties of epilayers and quantum wells of wide band gap II-VI materials, such as $\mathrm{ZnSe}$ on $\mathrm{GaAs} .^{3-5}$ In addition, the growth of II-VI materials in combination with III-V materials has a special appeal since one can, in principle, combine their attractive features in a single heterostructure. However, one has to contend with the lattice mismatch between the epilayer and the substrate. In this context, it is crucial to know the limiting conditions that lead to pseudomorphic growth by the accommodation of the strains due to lattice mismatch, rather than growth via the formation of misfit dislocations. The latter can have deleterious effects on the optical and electronic properties of a device. The compressibilites and/or thermal expansion coefficients of the materials forming the epilayer and the substrate are usually different. As a function of pressure and/or temperature, the lattice constants of the two constituent materials change differently leading to modifications in the biaxial strain experienced by them. Systems that are lattice matched at a certain pressure or temperature may become lattice mismatched as these parameters change and vice versa.

It has been shown that $\mathrm{ZnSe}$ epilayers grown pseudomorphically on GaAs exhibit the above effects from the photomodulated reflectivity (PR), ${ }^{6}$ photoluminescence (PL), ${ }^{7}$ and Raman-scattering ${ }^{8}$ studies under pressure. It was also shown that the tetragonal deformation potential $b$ is a function of either strain or volume deformation. $^{6,7}$ In this paper, we present a detailed PR and Raman study of a ZnTe epilayer grown on InAs.

\section{EXPERIMENT}

The epilayer sample consisted of a 63-nm epilayer of $\mathrm{ZnTe}$ grown on a $1.5-\mu \mathrm{m}$ InAs homoepitaxial epilayer on InAs by molecular-beam epitaxy. The high-resistivity $\mathrm{ZnTe}$ and InAs epilayers were grown in separate chambers. Cross-sectional TEM shows no dislocations or stacking faults in the epilayer or its interface, characteristic of pseudomorphic growth.

PR spectra were measured using a xenon arc source and a spectrometer dispersion of $3.2 \AA$. $0.02 \mathrm{~mW}$ of the $3638-\AA \mathrm{Ar}^{+}$laser line on a $200-\mu \mathrm{m}$ spot modulated at $200 \mathrm{~Hz}$ was used. Raman spectra were excited using $\sim 40 \mathrm{~mW}$ of various blue and violet lines of an $\mathrm{Ar}^{+}$ion laser focused to a spot size of $50 \mu \mathrm{m}$. Due to the near resonance with the $E_{0}$ gap, LO phonons up to third order were readily seen. High-pressure and piezomodulation and electromodulation techniques are discussed elsewhere. 9,10

\section{THEORETICAL CONSIDERATIONS}

A. Strains due to lattice mismatch

The built-in strains in the epilayer due to the lattice mismatch are characterized by

$$
\begin{aligned}
& \varepsilon=\varepsilon_{x x}=\varepsilon_{y y}=\frac{a_{s}-a_{e}}{a_{e}}, \varepsilon_{z z}=-2 \frac{c_{12}}{c_{11}} \varepsilon, \\
& \varepsilon_{x y}=\varepsilon_{y z}=\varepsilon_{z x}=0
\end{aligned}
$$


where $a_{s}$ and $a_{e}$ are the lattice constants of the substrate and the epilayer, respectively, and $c_{i j}$ are the elastic constants. The growth direction (001) is taken as the $z$ axis and $\varepsilon=\varepsilon_{x x}=\varepsilon_{y y}$ is referred to as the biaxial strain.

In the absence of strain, ${ }^{11}$ the heavy- and light-hole valence-band maxima at $\mathbf{k}=\mathbf{0}$ are degenerate. The biaxial strain shifts and splits the heavy- and light-hole band gaps $E_{g}^{\mathrm{hh}}$ and $E_{g}^{\mathrm{lh}}$ as follows:

$$
\begin{aligned}
& E_{g}^{\mathrm{hh}}=E_{g}+\left(\delta E_{h}+\delta E_{s}\right), \\
& E_{g}^{\mathrm{lh}}=E_{g}+\left(\delta E_{h}-\delta E_{s}\right)-\frac{2 \delta E_{s}^{2}}{\Delta},
\end{aligned}
$$

where $E_{g}$ is the fundamental gap and

$$
\begin{aligned}
& \delta E_{h}=2 a_{c v}\left(\frac{c_{11}-c_{12}}{c_{11}}\right) \varepsilon, \\
& \delta E_{s}=-b\left(\frac{c_{11}+2 c_{12}}{c_{11}}\right) \varepsilon .
\end{aligned}
$$

Here $a_{c v}$ is the combined hydrostatic deformation potential for transitions between the conduction and valence bands, $b$ is the shear deformation-potential constant, and $\Delta$ is the energy separation of the spin-orbit splitting. For an epilayer which has a lattice constant larger than that of the substrate, as is the case in $\mathrm{ZnTe} / \mathrm{InAs}$, the biaxial strain is compressive and a corresponding band-gap expansion occurs, with the heavyhole-derived band gap. Note that $\varepsilon$ is defined negative for biaxial compressive strain.

\section{B. Strains due to applied pressure}

Hydrostatic pressure decreases the lattice constants of a material. Since the compressibilities of different semiconductors vary, there can be pressure-induced biaxial strains between semiconductors that share a common interface. The strain thus generated can be formulated from the changes in the lattice constants. Murnaghan's equation of state $^{12}$ can be written as

$$
a(P)=a(1 \text { bar })\left[\frac{B^{\prime}}{B} P+1\right]^{-1 / 3 B^{\prime}},
$$

where $a(P)$ is the lattice constant as a function of applied hydrostatic pressure $P, B$ is the bulk modulus [ $\left.=\left(c_{11}+2 c_{12}\right) / 3\right]$, and $B^{\prime}$ is the pressure derivative of $B$. The pressure-induced strain $\varepsilon(P)$ from Eq. (3) is ${ }^{6,7}$

$\varepsilon(P)=\frac{a_{s}(P)-a_{e}(P)}{a_{e}(P)}$,

$\varepsilon(P)=\varepsilon(0)+\left(\frac{a_{s}}{a_{e}}\right)\left[\frac{P}{\left(c_{11}+2 c_{12}\right)_{e}}-\frac{P}{\left(c_{11}+2 c_{12}\right)_{s}}\right]$,

where the $c_{i j}$ 's are the elastic constants for the epilayer $(e)$ and the substrate $(s)$. In Eq. (4b), the initial latticemismatch strain is $\varepsilon(0)$ and only a first-order term in pressure is retained. If the bulk modulus of the epilayer is smaller than that of the substrate, as in our case, ${ }^{13}$ the pressure-induced stress is biaxially tensile. Since the ini- tial lattice mismatch yields biaxial compression, the heavy-hole-light-hole band splitting decreases with hydrostatic pressure. This is similar to $\mathrm{ZnSe} / \mathrm{GaAs}$. ${ }^{6,7}$

The pressure coefficient of the epilayer material in its bulk state is given by $\alpha_{\text {bulk }}=-a_{c v} / B$ and those for the light and heavy holes in the strained epilayer are $\mathrm{a}^{6,7}$

$\alpha_{\mathrm{hh}}^{\mathrm{lh}}=\alpha_{\text {bulk }}+\left(2 a_{c v} \frac{\left(c_{11}-c_{12}\right)}{c_{11}} \pm b \frac{\left(c_{11}+2 c_{12}\right)}{c_{11}}\right)_{e}\left(\frac{d \varepsilon}{d P}\right)$,

where the $+(-)$ sign in the second term is for light (heavy) holes.

The shift $\Delta \omega$ of the LO phonon of a strained epilayer from its bulk counterpart for (001) growth is ${ }^{14}$

$$
\frac{\Delta \omega}{\omega}=\frac{\widetilde{K}_{11}}{2} \varepsilon_{z z}+\frac{\widetilde{K}_{12}}{2}\left(\varepsilon_{x x}+\varepsilon_{y y}\right)=\left(\widetilde{K}_{12}-\widetilde{K}_{11} \frac{c_{12}}{c_{11}}\right) \varepsilon \text {, (6) }
$$

which are related to

$$
\begin{aligned}
& \widetilde{K}_{11}+2 \widetilde{K}_{12}=-6 \gamma_{\mathrm{LO}}, \\
& \gamma_{\mathrm{LO}}=\frac{B}{\omega_{\mathrm{LO}}} \frac{d \omega}{d P},
\end{aligned}
$$

where $\gamma_{\text {LO }}$ is the mode Grüneisen parameter. The hydrostatic and shear components of the stains in zinc-blende and diamond-type semiconductors are related to $\left(\widetilde{K}_{11}+2 \widetilde{K}_{12}\right)$ and $\left(\widetilde{K}_{11}-\widetilde{K}_{12}\right)$, respectively, for $(001)$ stress.

\section{RESULTS AND DISCUSSION}

We have measured the pressure dependence of the Raman-active LO phonon up to three orders for the ZnTe epilayer on InAs and bulk ZnTe, with both samples loaded in the same diamond-anvil cell. This has enabled us to directly measure the pressure dependence of the phonon shift $\Delta \omega$ of the epilayer with respect to that of the bulk. Figure 1 shows the first- and second-order Raman spectra at different pressures for the epilayer (dotted lines) and the bulk (solid lines). At 1 bar (not shown) the first-order LO phonon in the epilayer occurs at $\sim 1 \mathrm{~cm}^{-1}$ higher than that in the bulk. At the second and third orders, the difference is correspondingly larger. Under the applied pressure, the phonon peaks of the epilayer and the bulk approach each other and coalesce at $\sim 65$ kbars. This is evident from Fig. 1 and also in Fig. 2, in which the average separation $\Delta \omega$, normalized to the order, is shown. The mode Grüneisen parameter determined from $d \omega / d P$ of the phonon averaged over the three orders is

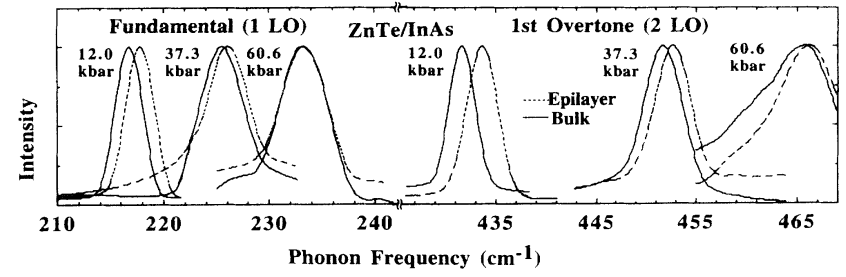

FIG. 1. The first- and second-order Raman spectra of the LO phonon of the ZnTe epilayer on InAs (dotted lines) and of bulk $\mathrm{ZnTe}$ (solid lines) at different pressures and $13 \mathrm{~K}$. 


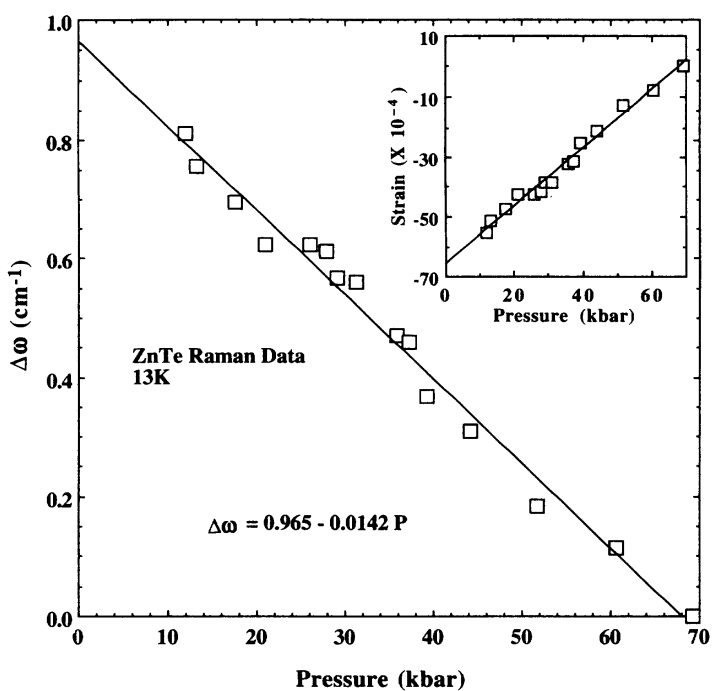

FIG. 2. The average separation of the LO phonon of the epilayer and bulk $\mathrm{ZnTe}$ vs pressure. The data from the first, second, and third orders are included. The inset shows the strain as a function of pressure obtained using Eqs. (6) and (7) (see text for details).

$\gamma_{\mathrm{LO}}=0.99 \pm 0.04$, for both epilayer and the bulk, in agreement with an earlier measurement. ${ }^{15}$ We also obtain values for $\widetilde{K}_{11}$ and $\widetilde{K}_{12}$ to be $(-2.3 \pm 0.3)$ and $(-1.7 \pm 0.3)$, respectively. No measured values of $\widetilde{K}_{i j}$ of $\mathrm{ZnTe}$ are available for comparison. Using the data from Fig. 2 and Eqs. (6) and (7), we determine that the strain in the $\mathrm{ZnTe}$ epilayer is linear with pressure (Fig. 2, inset). The solid line is a linear fit which yields the strain at 1 bar due to lattice mismatch, $\varepsilon=-65.8 \times 10^{-4}$ (in agreement with the value of $66.1 \times 10^{-4}$ from the known lattice constants ${ }^{13}$ ) and the pressure-dependent term $d \varepsilon / d P$ to be $0.961 \times 10^{-4} / \mathrm{kbar}$. The calculated strain using Eq. (4a) predicts a slight sublinearity; the data indicate that Eq. (4b), which contains only the linear term in pressure, is adequate.

Figure 3(a) shows the photomodulated-,
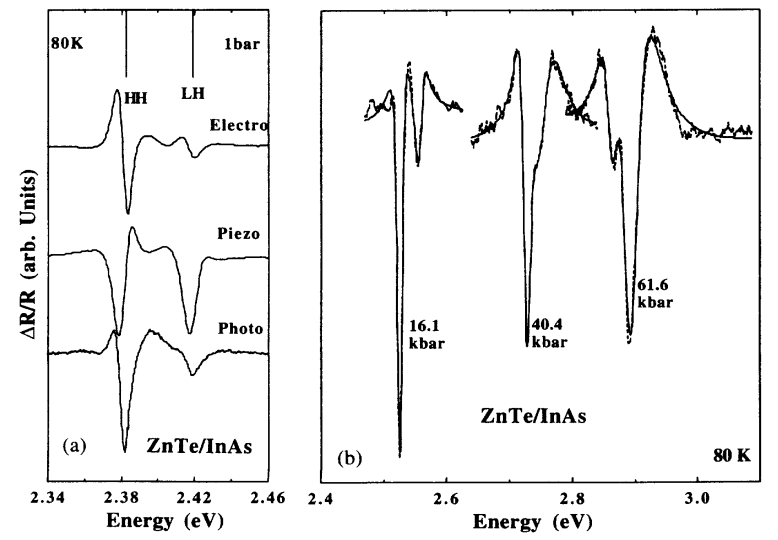

FIG. 3. (a) The electromodulated-, piezomodulated-, and photomodulated-reflectively spectra of the $\mathrm{ZnTe}$ epilayer at $\mathbf{8 0}$ $\mathrm{K}$. The two signatures correspond to the heavy- and light-hole excitons. (b) The photomodulated-reflectivity spectra of the $\mathrm{ZnTe}$ epilayer vs pressure. The dotted curves are the data and the solid curves are a fit to the data (see text). piezomodulated-, and electromodulated-reflectivity spectra of the $\mathrm{ZnTe}$ epilayer on InAs at $80 \mathrm{~K}$. The two signatures at 2.383 and $2.419 \mathrm{eV}$ correspond to the excitons associated with the heavy and light holes. Piezomodulation enhances the light hole despite its smaller matrix element. ${ }^{3,16}$

Figure 3(b) shows the PR spectra for the $\mathrm{ZnTe} / \mathrm{InAs}$ epilayer at $80 \mathrm{~K}$ for different applied pressures. The spectra are fitted as in Ref. 6.

Figure 4 shows the transition energies vs pressure. As pressure is increased, the heavy and light holes approach each other and cross at 55 kbars. Beyond 55 kbars, the fundamental gap changes from heavy to light hole. The crossing of heavy and light holes is analogous to that observed in pseudomorphic $\mathrm{ZnSe}$ on GaAs. ${ }^{6}$ PR signals could be observed only up to 62 kbars, where InAs undergoes a phase transition into the rocksalt structure.

Figure 4 shows that the $E_{g}^{\text {hh }}$ and $E_{g}^{\text {th }}$ transitions have a small sublinear behavior. The lines shown are fits to the functional form $E(P)=E(0)+\alpha P+\beta P^{2}$. The linear pressure coefficients are $\alpha_{\mathrm{hh}}=(8.96 \pm 0.20) \mathrm{meV} / \mathrm{kbar}$ and $\alpha_{\mathrm{lh}}=(9.45 \pm 0.20) \mathrm{meV} / \mathrm{kbar}$. The quadratic coefficients $\beta_{\mathrm{hh}}$ and $\beta_{\mathrm{lh}}$ are $(-0.013 \pm 0.002)$ and $(-0.034 \pm 0.003) \mathrm{meV} / \mathrm{kbar}^{2}$, respectively. The material parameters used in this paper ${ }^{13}$ are $c_{12} / c_{11}=0.574$, $B=517 \mathrm{kbars}$, and $B^{\prime}=4.7$ for $\mathrm{ZnTe}$; and $B=636 \mathrm{kbars}$ and $B^{\prime}=4.79$ for InAs. From the average of the $\alpha$ 's from Eq. (5), we can compute the hydrostatic deformation potential for $\mathrm{ZnTe}\left(\right.$ Ref. 16) to be $a_{c v}=-5.0 \pm 0.2 \mathrm{eV}$. We have also determined $a_{c v}$ from the volume deformation from Eq. (3) and the average band-gap energy as a function of pressure. ${ }^{7}$ The value thus obtained is also $-5.0 \pm 0.2 \mathrm{eV}$.

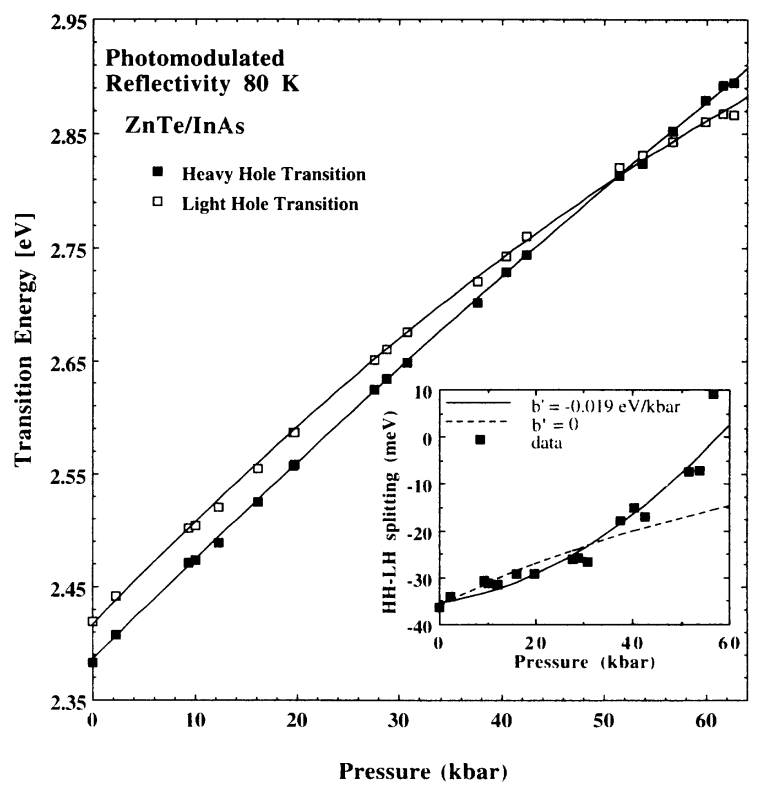

FIG. 4. The heavy- and light-hole transition energies vs pressure. The inset shows the separation between the heavy- and light-hole transitions energies. The dashed line is a fit using pressure-independent $b$ and the strain obtained from Eq. (3). The solid line in the fit is made by allowing the shear deformation-potential constant $b$ to be pressure dependent. 
The splitting $\left(E_{g}^{\mathrm{hh}}-E_{g}^{\mathrm{lh}}\right)$ as a function of pressure is shown in the inset of Fig. 4. Noting that

$$
E_{g}^{\mathrm{hh}}-E_{g}^{\mathrm{lh}}=2 \delta E_{s}+\frac{2 \delta E_{s}^{2}}{\Delta},
$$

from $\left(E_{g}^{\mathrm{hh}}-E_{g}^{\mathrm{lh}}\right)$ at 1 bar and the lattice-mismatch strain of $-6.58 \times 10^{-3}$ we find ${ }^{17} b=-1.27 \pm 0.05 \mathrm{eV}$ from Eqs. (1), (2d), and (8).

The pressure dependence of the splitting in Fig. 4 (inset) is clearly strongly superlinear. The strain-induced mixing of the light hole and the spin-orbit-split bands [second term in Eq. (8)] is too small to account for the measured sublinearity due to the large value of $\Delta[910$ $\mathrm{meV}$ at $300 \mathrm{~K}$ (Ref. 13)]. Hence $\left(E_{g}^{\mathrm{hh}}-E_{g}^{\mathrm{lh}}\right)$ vs pressure is determined primarily by $2 \delta E_{s}$, which depends on $b$, $c_{i j}$, and $\varepsilon(P)$.

The dotted curve in Fig. 4 (inset) is due to the strain calculated from Eq. (4a) and the value of $b$ obtained from the 1-bar data above. While the agreement is good up to 30 kbars, at higher pressures it indicates a slight sublinear rather than the observed superlinear pressure dependence. By allowing a pressure-dependent term $b^{\prime}$ ( $=d b / d P$ ) for $b$, the fit can be improved as shown by the solid curve. The values obtained from this fit are $b=-1.21 \mathrm{eV}$ and $b^{\prime}=-0.019 \mathrm{eV} / \mathrm{kbar}$.

A similar effect was observed in the case of $\mathrm{ZnSe}$ on GaAs. The Raman data $^{8}$ showed a linear pressuredependent strain whereas the PR and PL data ${ }^{6,7}$ gave evidence for a pressure-dependent shear deformation potential. Both the $\mathrm{ZnTe} / \mathrm{InAs}$ and $\mathrm{ZnSe} / \mathrm{GaAs}$ systems seem to indicate that the electronic bands in the epilayer deviate from the linear deformation-potential theory, whereas the lattice vibrations are in the linear regime.

We have considered other effects to explain the nonlinear splitting $\left(E_{g}^{\mathrm{hh}}-E_{g}^{\mathrm{lh}}\right)$ without invoking a pressuredependent $b$. Higher-order terms in the Murnaghan equation do not improve the fit in Fig. 4. A second-order term in volume deformation ${ }^{7}$ yields a value of $\eta=-b^{\prime} B=17.8 \mathrm{eV}$ per unit decrease of volume. This value is similar to that obtained in $\mathrm{ZnSe} / \mathrm{GaAs}$ and appears rather high. ${ }^{7}$

Both the Raman and PR data clearly show that the effect of applied pressure is to cancel the initial mismatch strain. The pressure $P_{m}$ at which lattice match occurs was found to be $\sim 55$ kbars from the PR data at which the splitting of the heavy- and light-hole bands goes to zero. In the Raman data, the difference in frequency of the Raman phonon of the epilayer and that in a separate bulk sample was compared. A somewhat higher $(\sim 65$ kbars) value of the observed $P_{m}$ is probably due to the uncertainty in the initial ( 1 bar) separation $\Delta \omega$. A decrease of $\sim 0.2 \mathrm{~cm}^{-1}$ in this value will bring the $P_{m}$ in agreement with the PR data.

\section{CONCLUSIONS}

Pressure tuning of the biaxial strain in pseudomorphic $\mathrm{ZnTe}$ on InAs is observed by Raman scattering (13 K) and photomodulated-reflectivity $(80 \mathrm{~K})$ studies. Values for the deformation-potential constants, mode Grüneisen parameters, and first-order strain derivatives of the LO phonon are determined. The Raman data show a linear dependence of the strain vs pressure. The PR data indicate that the shear deformation potential depends on pressure (or volume) in a manner similar to that observed in $\mathrm{ZnSe}$ epilayers on GaAs.

\section{ACKNOWLEDGMENTS}

The work by H.R.C. was supported in part by the U.S. Department of Energy under Contract No. DE-FG0289ER45402. M.C. was supported in part by the Research Corporation and A.R.O. Grant No. DAAL-03-91-G0381. A.K.R. and R.L.G. acknowledge support from the National Science Foundation (Materials Research Group No. DMR89-13706).
${ }^{1}$ M. A. Haase, J. Qiu, J. M. DePuydt, and H. Cheng, Appl. Phys. Lett. 59, 1272 (1991).

${ }^{2}$ H. Jeon et al., Appl. Phys. Lett. 59, 3619 (1991); 60, 2045 (1992).

${ }^{3}$ Y. R. Lee, A. K. Ramdas, L. A. Kolodziejski, and R. L. Gunshor, Phys. Rev. B 38, 13143 (1988).

${ }^{4}$ R. L. Gunshor et al., Appl. Phys. Lett. 50, 200 (1987); T. Yao et al., J. Cryst. Growth 81, 518 (1987).

${ }^{5}$ H. Mitsuhashi et al., Jpn. J. Appl. Phys. 24, L578 (1985); K. Ohkawa, T. Mitsuyu, and O. Yamazaki, Phys. Rev. B 38, 12465 (1988).

${ }^{6}$ B. Rockwell et al., Phys. Rev. B 44, 11307 (1991).

${ }^{7}$ J. A. Tuchman, S. Kim, Z. Sui, and I. P. Herman, Phys. Rev. B 46, 13371 (1992).

${ }^{8}$ L. J. Cui, U. D. Venkateswaran, B. A. Weinstein, and B. T. Jonker, Phys. Rev. B 44, 10949 (1991); in Frontiers of High Pressure Research, edited by H. D. Hochheimer and R. D. Etters (Plenum, New York, 1991), p. 269.

${ }^{9}$ A. Jayaraman, Rev. Mod. Phys. 55, 65 (1983); U. Venkateswaran et al., Phys. Rev. B 33, 8416 (1986); Y. M. Gupta and Z. A. Shen, Appl. Phys. Lett. 58, 583 (1991).

${ }^{10}$ R. G. Alonso et al., Phys. Rev. B 45, 1181 (1992).
${ }^{11}$ F. H. Pollak, in Strained-Layer Superlattices: Physics, edited by T. P. Pearsall (Academic, Boston, 1990), pp. 17-53.

${ }^{12}$ F. D. Murnaghan, Proc. Natl. Acad. Sci. U.S.A. 30, 244 (1944).

${ }^{13}$ Numerical Data and Functional Relationships in Science and Technology, edited by O. Madelung et al., Landolt-Börnstein, New Series, Group III, Vol. 17, Pts. a and b (Springer-Verlag, Berlin, 1982), and references therein.

${ }^{14}$ S. Ganesan et al., J. Ann. Phys. (N.Y.) 56, 556 (1970); E. M. Anastassakis et al., Solid State Commun. 8, 133 (1970); B. Jusserand and M. Cardona, in Light Scattering in Solids V, edited by M. Cardona and G. Guntherodt (Springer, New York, 1989), pp. 124-128 and 145.

${ }^{15}$ S. S. Mitra, O. Brafman, W. B. Daniels, and R. K. Crawford, Phys. Rev. 186, 942 (1969).

${ }^{16}$ H. Mathieu et al., Phys. Rev. B 36, 6581 (1987); Y. R. Lee et al., Proc. SPIE 794, 105 (1987); Appl. Phys. Lett. 50, 600 (1987).

${ }^{17}$ A. Blacha, H. Presting, and M. Cardona, Phys. Status Solidi B 126, 11 (1984). The values of $a_{c v}$ and $b$ measured for bulk $\mathrm{ZnTe}$ are -5.8 and $-1.8 \mathrm{eV}$, respectively. 